\title{
Vascular remodelling in intramyocardial resistance vessels in hypertensive human cardiac transplant recipients
}

\author{
John T Jenkins, Joseph J Boyle, Ian C McKay, David Richens, Allan R McPhaden, \\ George B M Lindop
}

\begin{abstract}
Objective-Cardiac transplant recipients often develop hypertension as a side effect of immunosuppressive treatment. The aim of this study was to use the serial endomyocardial biopsies taken to monitor rejection to study the early and sequential arterial changes in human myocardial resistance arteries as hypertension develops.
\end{abstract}

Methods-At least 14 biopsies were studied from each of 23 patients, divided into a normotensive group (12 patients with a diastolic pressure never greater than $90 \mathrm{~mm} \mathrm{Hg}$ ) and a hypertensive group (11 patients with more than $10 \%$ of diastolic pressure measurements above $100 \mathrm{~mm}$ Hg). Morphometric analysis of between 30 and 50 arteries and arterioles in two widely separated histological levels from each biopsy was undertaken using an Optomax image analyser.

Results-There was a correlation between blood pressure, particularly diastolic pressure, and rate of medial thickening of intramyocardial coronary resistance arteries and arterioles $(P=0.0025)$. There was also a correlation between serum cyclosporin $A$ concentrations and mean artery wall thickness $(P=0 \cdot 003)$.

Conclusions-Hypertension and cyclosporin A treatment are associated with significant wall thickening of intramyocardial resistance vessels in cardiac allograft recipients. These changes may be functionally and clinically important.

University of $\mathbf{G}$

Pathology, Westerm

and Royal Infirmaries,

Glasgow

J T Jenkins

J J Boyle

A R McPhaden

G B M Lindop

University of Glasgow

Department of

Immunology, Western

Infirmary, Glasgow

I C McKay

Scottish

Cardiopulmonary

Transplant Centre,

Royal Infirmary,

Glasgow

D Richens

Correspondence to:

Dr G B M Lindop

University of Glasgow

Department of Pathology,

Department of Pathology,

G11 6NT, United Kingdom.

Accepted for publication

24 January 1997

In hypertension, medial thickening is the earliest structural change in arteries. ${ }^{12}$ There is intense interest in its pathogenesis because medial thickening may give rise to an accentuated response to prevailing pressor stimuli-the "vascular amplifier" of early hypertension. ${ }^{3}$ Medial thickening with increased wall to lumen ratio could result from smooth muscle cell hypertrophy, hyperplasia, or increased overlap of the same number of smooth muscle cells of the same size (remodelling). These processes may occur in isolation or in concert, but their relative contributions remain uncertain. 45

Cardiac transplant recipients often become hypertensive largely because the immunosuppressive agents cyclosporin $\mathrm{A}$ and glucocorticoids raise blood pressure as a dose related side effect. ${ }^{6}$ Since serial endomyocardial biopsies are examined microscopically to monitor rejection, there is a unique opportunity to study the early and sequential arterial changes in human myocardial resistance vessels as hypertension develops. Diagnostic endomyocardial biopsies have been used to assess the thickness of intramyocardial arteries in essential hypertension by morphometry. ${ }^{78}$ Similar studies have not been undertaken in the cardiac transplant population, nor has hypertensive vascular disease been studied longitudinally with time, either in animals or in man. We elected to use serial cardiac allograft biopsies to quantify the changes in arterial geometry that occur in the early phases of hypertension in man.

\section{Methods}

PATIENTS

Twenty three orthotopic cardiac transplant recipients were selected from the files of the Scottish Cardiopulmonary Transplant Centre. To maximise the separation in blood pressure we selected 11 patients with diastolic blood pressure that was often (more than $10 \%$ of measurements) more than $100 \mathrm{~mm} \mathrm{Hg}$, and 12 patients with diastolic blood pressure never more than $90 \mathrm{~mm} \mathrm{Hg}$. The former group was considered to be hypertensive and the patients were treated as clinically indicated; the latter group was considered to be normotensive. They accounted in total for approximately half of the transplant recipients at this centre. The two groups of patients were matched for age, sex, number and severity of rejection episodes, and immunosuppressant drug treatment. Also available were monthly blood pressure measurements, doses of glucocorticoids, serial cyclosporin A serum concentrations, and donor age and sex.

\section{TISSUE PROCESSING AND MORPHOMETRIC} ANALYSIS

Endomyocardial biopsies were fixed by immersion in formalin, processed into paraffin wax, and sectioned at $4 \mu \mathrm{m}$. Sections were coded by random numbering to ensure ignorance of their temporal sequence and patient details. All arteries and arterioles were measured by one observer (J) provided they satisfied the following criteria: a complete layer of smooth muscle cells in the media; a visible lumen; and no histological abnormalities or artefacts. Vessels were 
Figure 1

Photomicrograph of a cross section of a small intramyocardial coronary artery. The coordinate points used for artery measurement are superimposed on the artery. Haematoxylin and eosin $\times 250$.

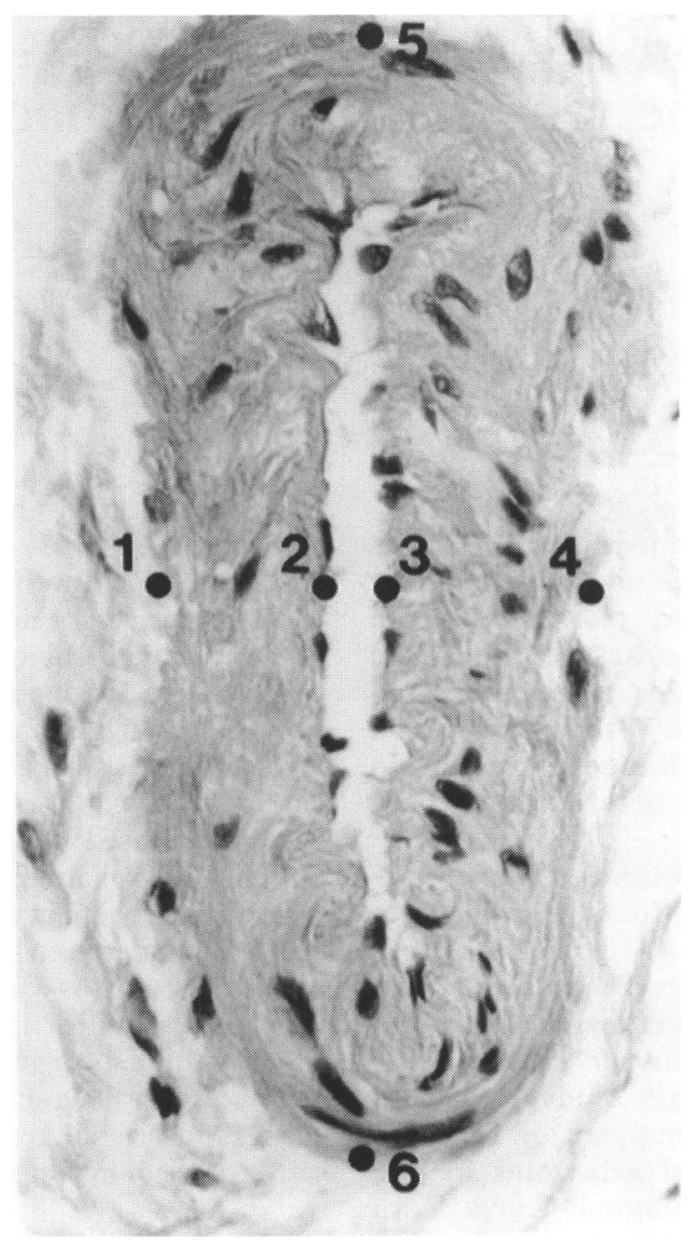

excluded if they were situated in regions of rejection, scarring, previous biopsy site, organising thrombus, or epicardial fat. Between 30 and 50 arteries and arterioles from two widely separated histological sections from a minimum of 14 biopsies from each of the patients were subjected to morphometric analysis. This was carried out using an Optomax image analyser (AMJ) interfaced to an IBM PS2 computer run-

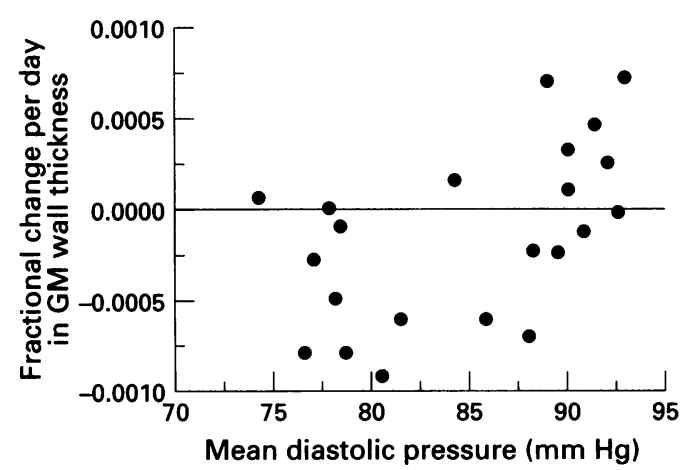

Figure 2 Scattergram in which the $x$ axis represents the diastolic blood pressure (DBP) for each patient, averaged over the course of the study. The $y$ axis shows the rate of change in log mean wall thickness of each patient's change in log mean wall thickness of each patient's of the regression line which best fitted the scattergram of each biopsy's log mean wall thickness with time. The negative fractional changes imply a net thinning of resistance vessel walls, predominating at lower values of DBP. Positive fractional changes imply that the vessels become thicker with this predominating at higher values of DBP. One outlying point that has the lowest of both values has been omitted from the graph in order to avoid inconvenient expansion of the scale.

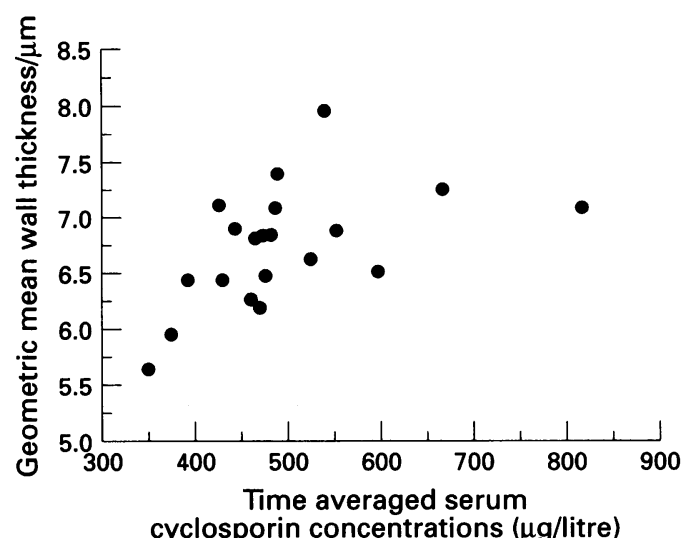

Figure 3 In this scattergram the $x$ axis shows each patient's serum cyclosporin level averaged over the duration of the study. The $y$ axis shows each patient's logarithmic (geometric) mean wall thickness over all biopsies given in micrometres $(\mu \mathrm{m})$. The wall thickness positively correlates with time averaged cyclosporin concentrations (Spearman's $p=0.627, P=0.003$ ).

ning Vids-V software and employing a previously described modification of the method of Short. ${ }^{9}$ This enabled calculation of mean wall thickness and log wall thickness. The following indices were further derived: gradient of log wall thickness with time; gradient of log wall/lumen ratio with time; and gradient of external diameter with time.

\section{STATISTICAL METHODS}

The relations between blood pressure and morphometric indices were measured and tested by Spearman's rank correlation method. Where several correlation analyses were carried out on the same set of data, compensation for the multiple tests was made by choosing a more stringent test criterion based on the Bonferroni inequality.

\section{Results}

HISTOLOGICAL APPEARANCES

An example of an intramyocardial artery is shown in fig 1 . The coordinates measured are shown superimposed on this artery. In the hypertensive patients the arteries and arterioles showed medial thickening but the intima was always normal. Hyalinisation was never observed.

\section{BLOOD PRESSURE}

There was a significant difference in diastolic blood pressure between the two groups (Student's $t$ test, $\mathrm{P}=0.01$; data not shown). The hypertensive patients showed little change and little individual variation in blood pressure with time, whereas the blood pressures of the normotensive group were more variable.

BLOOD PRESSURE CORRELATIONS WITH MORPHOMETRIC INDICES

The walls of arteries and arterioles of the high pressure group tended to become thicker with time, whereas those of the normotensive patients tended to become thinner (fig 2), the difference between the gradients of the log wall thickness of the two groups being of marginal 
Parametric (Pearson's) and non-parametric (Spearman's) correlation coefficients between various pharmacological, morphometric, and haemodynamic variables

\begin{tabular}{|c|c|c|c|c|}
\hline & CSP mean & Log mean $W$ & $\begin{array}{l}\text { Mean } \\
\text { systolic }\end{array}$ & $\begin{array}{l}\text { Mean } \\
\text { diastolic }\end{array}$ \\
\hline \multicolumn{5}{|c|}{ Pearson's product-moment correlation coefficients: } \\
\hline Log mean W & $0 \cdot 518$ & & & \\
\hline Mean systolic & $-0 \cdot 152$ & 0.415 & & \\
\hline Mean diastolic & -0.075 & $0 \cdot 231$ & $0 \cdot 747$ & \\
\hline MAP & $-0 \cdot 114$ & 0.333 & 0.917 & 0.950 \\
\hline \multicolumn{5}{|c|}{ Spearman's rank correlation coefficients: } \\
\hline Log mean W & 0.618 & & & \\
\hline Mean systolic & -0.072 & $0 \cdot 254$ & & \\
\hline Mean diastolic & $-0 \cdot 140$ & $0 \cdot 115$ & $0 \cdot 812$ & \\
\hline MAP & -0.123 & $0 \cdot 236$ & 0.922 & 0.954 \\
\hline
\end{tabular}

CSP mean, time averaged serum cyclosporin concentration (calculated as in fig 3 legend); Log mean W, logarithmic mean of wall thickness over all of the patient's biopsies; Mean systolic, arithmetic mean systolic blood pressure taken over all of each patient's recordings; Mean diastolic, arithmetic mean diastolic blood pressure taken over all of each patient's recordings; MAP, arithmetic mean arterial pressure.

Each correlation coefficient was based on a scattergram (not shown) analogous to those in figures 2 and 3 in which each patient is represented by one point. CSP mean correlated posifigures 2 and 3 in which each patient is represented by one point. CSP mean correlated posiindices of blood pressure. suggesting that useful information can be obtained from them. ${ }^{10}$ Other workers have reached the same conclusion. ${ }^{?}$

\section{EFFECT OF BLOOD PRESSURE ON VESSEL WALI THICKNESS}

We have shown that raised blood pressure is associated with medial thickening of intramyocardial coronary arteries and arterioles in cardiac allografts. This agrees with current concepts of the effects of blood pressure on resistance vessels ${ }^{124}$ and with the observed effects of essential hypertension on intramyocardial arteries and arterioles in biopsies of native hearts. ${ }^{78}$ Artery wall thickening correlated best with diastolic blood pressure, less strongly with mean arterial pressure, and not with systolic blood pressure. This finding is in keeping with the view that intramyocardial coronary resistance arteries are protected from the effects of systolic blood pressure because there is no flow through them during systole. ${ }^{2}$ The weaker correlation with mean blood pressure compared with diastolic pressure could be due to a diminution of the correlation from the contribution of systolic blood pressure to the mean arterial pressure value. Overall, our data suggest that raised diastolic pressure causes thickening of small intramyocardial coronary arteries and that these arteries are protected from the effects of systolic blood pressure.

Hyaline arteriolosclerosis of intramyocardial coronary arteries was not observed in the hypertensive group. This is in agreement with previous studies of arteriolosclerosis. ${ }^{2}$ The development of arteriolosclerosis may be more dependent on the height of systolic pressure than of diastolic blood pressure; alternatively the development of medial thickening could be more sensitive than arteriolosclerosis to pressure. Non-immune binding of plasma proteins, including complement, to proteoglycans in the vessel wall has also been suggested as a possible cause of arteriolosclerosis. ${ }^{11}$ Histologically visible arteriolosclerosis may require a prolonged build up of plasma proteins in the vessel wall; however, the time course of this study was only 15 months, and this period may be too short. The inability to produce hyaline in the resistance arteries of either diabetic or hypertensive animals (personal observation) is in accord with the latter explanation.

The mechanisms underlying the reduction of vessel wall thickness in the normotensive group are unclear. Candidate causes include: cardiac denervation; regression of pre-existing hypertensive changes in donor arteries; and resolution of arterial damage resulting from ischaemia or rejection. Unfortunately, blood pressure readings recorded in the donors before their terminal illness were not usually available. However, blood pressure differences between donor and recipient may clearly affect the ultimate geometry of cardiac resistance vessels.

EFFECTS OF DRUG TREATMENT ON VESSEL THICKNESS

Average serum cyclosporin A concentrations showed a strong correlation with arterial wall thickness (fig 3). Since cyclosporin is an estabto cause tissue shrinkage and distortion. We have estimated that there is a $10 \%$ linear distortion of small arteries due to sectioning of paraffin embedded endomyocardial biopsies, 
lished cause of systemic hypertension, a possible pathogenic mechanism is immediately apparent. ${ }^{6}$ However, our statistical analysis suggests that any effect of cyclosporin on artery structure is unlikely to have been mediated by hypertension. Therefore, either direct or immunological actions of cyclosporin on both vascular smooth muscle and endothelial cells may be important in mediating vessel thickening. Recent experimental work in heterotopic rat cardiac allografts suggests that cyclosporin inhibits the intimal thickening seen in large artery transplant associated coronary artery disease. ${ }^{12}$ The effects of cyclosporin on the small subendocardial arterioles examined in this study are likely to have a different pathogenesis, since there were no intimal changes and there was no histological evidence that arterial thickening was related to rejection. Cyclosporin may directly affect vasoconstriction or artery growth. There is evidence that it may increase medial reactivity in liver transplant recipients. ${ }^{13}$ In vitro studies show that candidate mechanisms include enhanced contractile responses to angioten $\sin ^{1415}$ and platelet derived growth factor ${ }^{15}$; increased free intracellular calcium and agonist induced inositol polyphosphate ${ }^{15}$; diminished relaxation in response to nitric oxide generation ${ }^{16}$; and reduced induction of nitric oxide synthase. ${ }^{17}$

It is likely that the aggressive antihypertensive drug regimen employed in cardiac transplant recipients may have reduced the overall degree of medial thickening in the hypertensive group. If so, it may account for the lack of statistically significant differences in the morphometric indices between the hypertensive and normotensive groups.

\section{CLINICAL IMPLICATIONS}

Intramyocardial arterial and arteriolar thickening, as observed in this study, is likely to have functional implications and may contribute to cardiac allograft ischaemia. For example, thickening of the walls of intramyocardial coronary arterioles in hypertension correlates with increased minimum coronary resistance and reduced coronary vasodilator reserve. ${ }^{8}$ Furthermore, we have shown that measurable arterial thickening occurs despite aggressive post-transplant antihypertensive treatment. New treatments may have to be considered in order to inhibit these small vessel changes if they prove to be clinically important.

1 Folkow B. Physiological aspects of primary hypertension Physiol Rev 1982;62:348-79.

2 Lindop GBM. The effects of hypertension on the structure of human resistance vessels. In: Swales J D, ed. Textbook of hypertension. London: Blackwell Scientific Publications, 1994:663-70.

3 Lever AF. Slow pressor mechanisms in vascular hypertrophy: a role for hypertrophy of resistance vessels. $f$ Hypertens 1986;4:515-24.

4 Gibbons GH, Dzau VJ. Emerging concept of vascular remodelling. $N$ Engl ₹ Med 1994;330:1431-8.

5 Mulvany MJ. The development and regression of vascular hypertrophy. F Cardiovasc Pharmacol 1992;19(suppl 2): h22-7.

6 Thompson ME, Shapiro AF, Johnson AM, Reeves R Itzkoff J, Ginchereau E, et al. New onset hypertension following cardiac transplantation: a preliminary report and analysis. Transplant Proc 1983;15:2573-7.

7 Schwartzkopff B, Motz W, Knauer S, Frenzel H, Strauer BE. Morphometric investigation of intramyocardial arterioles in right septal endomyocardial biopsy of patients with arterial hypertension and left ventricular hypertrophy. $\mathcal{F}$ Cardiovasc Pharmacol 1992;20(suppl 1):S12-17.

8 Schwartzkopff B, Motz W, Frenzel H, Vogt M, Knauer S, Strauer BE. Structural and functional alterations of the intramyocardial arterioles in patients with arterial hypertension. Circulation 1993;88:993-1003.

9 Short D. Morphology of the intestinal arteriole in chronic human hypertension. Br Heart $\mathcal{f} 1966 ; 28: 184-92$.

10 Boyle JJ, Jenkins J, McKay IC, McPhaden AR, Lindop EBM. An assessment of the distortion of arteries due to sectioning in endomyocardial biopsies. F Pathol 1997; 181:243-6.

11 Gamble CN. The pathogenesis of hyaline arteriolosclerosis. Am ₹ Pathol 1986;122:410-20.

12 Paul LC, Davidoff A, Benediktsson H. Cardiac allograft atherosclerosis in the rat: the effect of histocompatibility factors, cyclosporin and an angiotensin converting factors, cyclosporin and an angiotensin conve

13 Potocnik SJ, Phillips PA, Hardy KJ. Human resistance artery reactivity is altered by liver transplantation and artery reactivity is altered by liver transplantation and
treatment with cyclosporin A. Transplant Proc 1992;24: treatment

14 Auch-Schwelk W, Bossaller C, Gotze S, Thelen J, Fleck E. Endothelial and vascular smooth muscle function after chronic treatment with cyclosporin A. $\mathcal{f}$ Cardiovasc Pharmacol 1993:21:435-40.

15 Locher R, Huss R, Vetter W. Potentiation of vascular smooth muscle cell activity by cyclosporin A. Eur $\mathcal{F}$ Clin Pharmacol 1991;41:297-301.

16 Verbeke $M$, Van de Voorde J, de Ridder L, Lameire $N$. Functional analysis of vascular dysfunction in cyclosporin treated rats. Cardiovasc Res 1994;28:1152-6.

17 Marumo T, Nakaki T, Hishikawa $K$, Suzuki $H$, Kato $R$, Saruta $T$. Cyclosporin A inhibits nitric oxide synthase induction in vascular smooth muscle cells. Hypertension 1995;25:764-8. 